\title{
A study to see effects of radiation alone and radiation with concurrent chemotherapy for advanced squamous cell carcinoma of the head and neck
}

\author{
Bhattacharjee $\mathrm{B}^{1}$, Sengupta $\mathrm{AK}^{2}$, Bari $\mathrm{MA}^{3}$, Hai MA ${ }^{4}$
}

\begin{abstract}
Aim : To observe the responses of radiation with concurrent chemo-radiation and radiation alone at the primary tumour sites and metastatic neck nodes of patients with head and neck cancers weekly during treatment, after completion of treatment and at 6 weeks interval after treatment. Methods : Randomizes prospective study was carried out in the Department of Radiotherapy, Dhaka Medical College and Hospital, Dhaka during the period of July 2003 to June 2004. Patients with histologic and/or cytologic confirmation of the diagnosis of squamous cell carcinoma of head and neck without distance metastasis were included in this study. After proper evaluation Arm-A was treated by concurrent chemotherapy with Inj. Cisplatinum $75 \mathrm{mg} / \mathrm{m} 2$ on Day-1, Day-21 and Day-42 and conventional radiotherapy with a total dose of $60 \mathrm{~Gy}$ in 30 fractions in 6 weeks. Arm-B was treated by conventional radiotherapy with the same dose as that of Arm-A. Every patient was evaluated by monitoring routinely to see the responses. All the resultant informations were kept preserved on a prescribed data sheet. These informations were than analyzed statistically and results were compiled accordingly. Result : Out of 28 patients in Arm-A, complete desirable response was observed in 19 cases and partial response in 9 cases, where as out of 30 patients in Arm-B it was 7 and 23 cases respectively. Conclusion: Concurrent chemo-radiation showed better response than radiation alone, though toxicities were little bit higher but it was acceptable and easily manageable.
\end{abstract}

\section{Introduction}

"Head and Neck Cancer" is the name given to a variety of malignant tumours that occur in the head and neck region excluding tumors that occur in the brain. There is no complete statistics of head and neck cancer in our country like the devolped countries, but according to some Institution-based study done in 1990 among 3399 new cancer patients, attending the Department of Radiotherapy, Dhaka Medical College Hospital, revealed head and neck cancer is about $33.15 \%$ of all malignancies in male, $20.78 \%$ in female and $28.68 \%$ in all malignancies in both sexes 1 . The vast majority of head and neck neoplasms arise from surface epithelium and are, therefore, squamous cell carcinomas (about $90 \%$ ) or one of its many variants including lymphoepithelioma, spindle cell carcinoma, verrucous and undifferentiated carcinoma. Unfortunately in our country, most of the patients with head and neck cancer attend the Radiotherapy

Department in advanced stage. This may be due to ignorance, poverty, faulty referral system, illiteracy and with some traditional false belief.
1. Dr. Biswajit Bhattacharjee, Consultant KYAMCH Cancer Centre, 2. Dr. Ashim Kr. Sengupta, Assistant Professor, KYAMCH Cancer Centre, 3. Dr. M. A. Bari, Consultant KYAMCH Cancer Centre, 4. Prof. (Dr.) M. A. Hai, Professor \& HOD KYAMCH Cancer Centre Now a days, it is claimed that cancer is curable due to modern diagnostic tools and multi-modal treatment as demonstrated in numerous clinical trails, investigations and new knowledge in basic research2.

Standard treatment for head and neck cancers consists of surgery and/or radiotherapy. However, standard treatment alone is less successful for advanced head and neck cancer3. A variety of radiation techniques are available and the appropriate choice for an individual patient must be made. External megavoltage irradiation is the corner stone of modern treatment in most patients

\section{Methods}

A total of 58 patients with head and neck cancer were selected randomly by maintaining the patient selection criteria. After selection every patient was asked to give informed written consent regarding study and treatment. 
All patients were interviewed and informations were documented in a prescribed data collection form. A thorough clinical examination was done for each patient including relevant investigations such as complete blood count, liver function test, kidney function test, Xray chest $\mathrm{P} / \mathrm{A}$ view, lateral view $\mathrm{X}$-ray of the soft tissue neck etc. along with indirect laryngoscopy and results were recorded. The clinical staging was done according to TNM classification. Stage III and IV, locally advanced, without distant metastasis were treated.

Chemotherapy : Cisplatin was administered $75 \mathrm{mg} / \mathrm{m} 2$ rather than standard 80 to $100 \mathrm{mg} / \mathrm{m} 2$ in intravenous drip on Day-1, Day-21 and Day-42 for three doses during radiotherapy. Proper hydration (pre and post chemotherapy) was maintained during the treatment period. Complete blood count was carried out before each dose of chemotherapy.

Radiotherapy : Radiotherapy was delivered by using a tele-cobalt unit in all patients (both Arm-A and Arm-B) in the study. All the patients were treated by parallel opposed fields and the radiation dose was calculated in the central axis at the middle of the two opposing fields. Conventional fractionation (200cGy per fraction, one fraction per day, five days per week) was used. Before irradiation, dental examination was performed carefully. Instructions for oral hygiene were given to prevent dental decay and to reduce the risk of subsequent bone necrosis. After verification of field arrangements and inspection of the dose distribution, the treatment prescription was finalized and signed. Target volume was decided clinically and radiographically. Treatment volume was then decided taking approximately $1-2 \mathrm{~cm}$ generous margin around the target volume. In presence of metastatic neck node in a close proximity to the primary lesion, the treatment volume was adjusted and the node was included in the same field. Treatment area was marked on the skin with gention violate placing the patient on the table. Patient's position was correlated with the position of the patient at the machine during treatment. Anatomical landmarks and the field size were noted on the radiation therapy card, which helped to remark on the skin of treatment volume when necessary.

Patients of both groups were reviewed every week up to the completion of treatment and findings of local examination (tumour and/or nodal regression) were recorded and compared with the previous findings and documented on sheet.

Standard criteria for complete response (CR) and partial response (PR) were used. $\mathrm{CR}$ and $\mathrm{PR}$ were defined as complete regression and greater than $50 \%$ regression of clinically measurable disease respectively.

Toxicities to chemo-radiation and radiation alone were carefully recorded as per common toxicity criteria by Fischer et al. 1994. Supportive treatment required for those toxicities were given accordingly. After completion of treatment, all the patients were advised to come for follow-up examination after every 6 weeks.

All the observations and results were then statistically analyzed. Differences in initial response rates were evaluated by Chi-square test.

\section{Results}

Total 58 patients of head and neck carcinoma were studied in two arms Arm-A was the concurrent chemoradiotherapy group containing 28 patients and Arm-B included 30 patients who received radiotherapy alone. Majority of the patients were males, 45 out of 58 and oral cavity and oropharynx was the common site. Most of the patients were between 40 to 49 years age group. Histologically maximum patients were poorly differentiated. In this study shows that chemoradiotherapy was very effective in stage III and stage IV disease and moderately significant in poorly differentiated histology. It also shows that chemoradiotherapy response was significant where the primary site was larynx. Treatment toxicities were found more in Arm-A than in Arm-B.

In post-treatment evaluation, Arm-A showed better result with complete response in 19 patients and partial response in 9 cases, whereas in Arm-B, it was 7 and 23 cases respectively.

\section{Discussion}

In this study, it has been observed that head and neck carcinoma occurred in patients with a male and female ratio 3.62:1 in both groups. Average age of the patients during thesis time in Arm-A and Arm-B groups 52.4 years. These observations correlate with Bomford and Jennings.4,5 They have also found that this is a disease of advancing age and affecting male gender more.

Regarding the sites of involvement in both groups ( $\mathrm{n}=$ 58), this study showed larynx and oral cavity \& oropharynx were the major primary sites. Oral cavity 
and oropharynx was involved in 20 (34.48\%) patients and in another $20(34.48 \%)$ patients larynx was involved. Other sites of involvement in this study were hypopharynx in 12 patients (20.68\%) and nosopharynx in 6 patients $(10.34 \%)$ in both groups. Glottic and supraglottic regions were involved in the laryngeal primary disease. Among the oral and oropharyngeal cancers tongue, tonsil and retro-molar trigone were the common sites, pyriform fossa was the common site in hypopharyngeal cases. These findings regarding the sites of involvement are similar to the study by Zagars et al. and Bomford et al.6,4

The majority of the patients entered in this study belongs to low to middle class socioeconomic condition and a total of 51 patients $(87.93 \%)$ contribute this, whereas a total of $7(12.07 \%)$ came from high socioeconomic class. The reason for more number of low to middle class patients was may be due to that, higher class patients can go to private sector or to abroad to get treatment by cost of money which is not possible for low to middle class patients.

About their habit, it was observed that tobacco use either in the form of chewing or in the form of smoking was common in $85 \%$ cases in both the groups, 9 (15\%) patients were found that they didn't have such type of habits. Jennings described tobacco use as the greatest risk factor5.

After clinical staging, it has been observed that 40 (68.97\%) patients from the both group was in stage III and rest of the patient that is $18(31.03 \%)$ patients were in stage IV disease. To find out loco-regional control after treatment, stage III disease in Arm-A was compared with stage III diasease in Arm-B and so on. After statistical analysis with Chi-square test, the result was found significant in stage III and stage IV (more advanced) that means that the efficacy of chemoradiotherapy was more in case of advanced disease.

Al-Sarraf et al.7 in their study decsriberd that complete response was $82 \%$ in stage III and $66 \%$ in stage IV. Here in this, I found $73.68 \%$ complete response in stage III and $55.56 \%$ complete response in stage IV disease with concomitant chemo-radiotherapy. This difference might be due to some limitations during treatment time. But Gasparini et al. have seen that chemo-radiotherapy offers almost similar result in stage III and stage IV head and neck cancers. The better loco-regional control with concomitant chemo-radiotherapy may be due to the fact that local anti-tumour activity of radiation is enhanced due to simultaneous use of chemotherapeutic agent like cisplatinum as radiation sensitizer8.

In this study, all the lesions were squamous cell carcinoma of different types of cellular differentiation. This study revealed very significant result $(\mathrm{P}<0.01)$ in poorly differentiated histology group. A preliminary report from AL-Sarraf et al. (1981) suggested that poorly differentiated tumours respond better than well differentiated tumours. In this study, poor, well and moderately differentiated tumours complete response was $84.62,62.5$ and $42.86 \%$ respectively. This study correlates to study by Al-Sarraf et al.7

Regarding the topographical involvement of the disease, the response was found significant $(\mathrm{P}<0.05)$ where the primary site was larynx. Apart from this study, Gasparini et al. and Lin et al. described that there is no significant difference in response with chemoradiotherapy in relation to topographical involvement9,10. Karp et al. proved that larynx preservation is possible with chemo-radiotherapy. In his study, he found 33 of 35 patients (94\%) with carcinoma larynx were able to retain their larynx for the remainder of their lives11.

In this study (both Arm-A and Arm-B groups), presenting complaints of most of the patients were pain in throat and/or oral cavity in 20 patients $(34.48 \%)$ of the total population $(n=58)$, neck node swelling in 17 patients (29.31\%), hoarseness of voice in 17 patients (29.31\%), dysphagia and referred otalgia in $3(5.17 \%)$ and $1(1.72 \%)$ respectively. In Arm-a, mean duration of time to get relief from major presenting complaints was 17.4 days and in Arm-B was 19.6 days.

Overall response with concomitant chemo-radiotherapy (Arm-A) and radiotherapy alone (Arm-B) in this study revealed that complete and partial response in Arm-A were $67.86 \%$ and $32.14 \%$ and in Arm-B were $23.33 \%$ and $76.67 \%$ respectively. Statistical analysis with Chisquare test was performed and $\mathrm{P}$ value was determined as $\mathrm{P}<0.01$. So, better loco-regional control was observed in Arm-A group than Arm-B group. Some other studies with concomitant chemo-radiotherapy in head and neck cancer patients also observed significant results. Macmillan et al. and Lin et al. found 84 and $77.4 \%$ complete response respectively 12,10 . In a study by Dinges et al.found that complete response with concomitant chemo-radiotherapy for locally advanced 
head and neck cancer was $76 \% 13$.

Regarding the treatment acute toxicities, mucositis was common usually found in third week 4. Patients in Arm-A developed grade II and grade III mucositis more than in Arm-B group. Skin reaction was observed increased in Arm-A than in Arm-B. Nausea and vomiting were less reported in Arm-B but little more in case of Arm-A. Haematological toxicities were observed in Arm-A but none in Arm-B. The toxicities developed were managed accordingly.

The very significant loco-regional tumour control in head and neck cancer with concomitant chemo-radiotherapy may be supported by observing the survival rate. Although most of the studies in abroad by Wendt et al. Lin et al. and Munro et al. found significant survival rates with chemoradiotherapy but here, this could not be carried out due to shorter duration of study14,10,15. In this study, all the patients were followed-up regularly and were found well up to the last follow-up.

The result in this study may not be same with another study because other factors like age, nutritional status, heterogeneity of head and neck cancer presentation, duration of study, dosage, sample size and multi-center participation may clearly affect the data.

In conclusion, it may be said that stage III and stage IV (advanced) poorly differentiated laryngeal cancer in patients having UICC grade-I performance status is highly responsive to concurrent chemo-radiotherapy than radiotherapy alone.

\section{References}

1. Sarma SK, Akhter PS, Alam AMMS, Ansary HR, Uddin M. 1992. Distribution pattern of 3399 new cancer patients: A one year study. Bangladesh Med J XXV.

2. Rubin P, Cooper RA. 1993. Statement of the clinical oncologic problem, In: Rubin P (ed.).Clinical Oncology: A multidisciplinary Approach for Physician and Students. 7th ed. WB Saunders Company, Philadelphia. pp.1-12.

3. Tsukuda M. 1995. Neo-adjuvant chemotherapy in patients with head and neck cancer: Gan toKagaku Ryho, Nov 22:13;1905-11.

4. Bomford CK, Kunkler IH, Sherrif SB, Miller H. 1993. Cancer chemotherapy, In: Walter and Miller's Textbook of Radiotherapy, Radiation Physics, Therapy and Oncology, 5th ed. Churchill Livingstone, Edinburgh. Pp. 530-9.

5. Jennings RL. 1997. Cancer of the head and neck, In:
Gates RA, Fink RM (eds.). Oncology Nursing Secrets. Jaypee Brothers, New Delhi. Pp.180-7.

6. Zagars GK, Norante JD, Smith JL, McDoland S. 1993.

Tumours of the head and neck, In: Clinical Oncology: A multidisciplinary Approach for Physician and Students. 7th ed. WB Saunders Company, Philadelphia. pp.319-62.

7. Al-Sarraf M, Drelichman A, Jacobs J, 1981. Adjuvant chemotherapy with cisplatin, oncovin and bleomycin followed by surgery and/or radiotherapy in patients with advanced previously untreated head and neck cancer: Final report, In: Jones SE, Salmon SE (eds.) Adjuvant therapy of cancer. III. Grune and Stratton, Philadelphia. 145-52.

8. Gasparini GP, Recher GF, Testolin A, Fier SD, Pannizoni GA, Cristoferi V, et al. 1992. Synchronus radiotherapy and chemotherapy with cisplatin in the management of locally advanced or recurrent head and neck cancer. Am J Clin Oncol 15:242.

9. Perry MC. 1992. The Chemotherapy Source Book, 1st ed. Williams and Wilkins. pp.924-6.

10. Lin JC, Jan JS, Hsu CY. 1994. Concomitant chemoradiotherapy for advanced head and neck Cancer. Jpn J Clin Oncol 24:94-100.

11. Karp DD, Vanghan CW, Carter R, Willett B, Heeren T, Calarese P, et al. 1991. Larynx preservation using induction chemotherapy plus radiation therapy as an alternative to laryngectomy in advanced head and neck cancer: A long time follow up report. Am J Clin Oncol $14: 273-9$

12. MacMillan CH, Carrick K, Bradley PJ, Morgan AL. 1991. Concomitant chemo/radiotherapy for advanced carcinoma of the head and neck. Br J Radiol 64:941-6.

13. Dinges S, Budach V, Stuschke M, Budach W, Boehmer D, Schrader M, et al. 1997. Chemo-radiotherapy for locally advanced head and neck cancer: long term results of phase II trial. Eur J Cancer 33:1152-5.

14. Wendt TG, Grabenbauer GG, Rodel CM, Thiel HJ, Aydin H. 1998. Simultaneous radio-chemotherapy versus radiotherapy alone in advanced head and neck cancer: A randomized multicenter study. J. Clin Oncol 16:1318-24.

15. Munro AJ. 1995. An overview of randomized controlled trials of adjuvant chemotherapy in head and neck. Br J Cancer 71:83-91.

16. Bangladesh Bureau of Statistics 2003. Report of house hold income and expenditure survey, December 2000 to March 2003. Ministry of Planning, Dhaka, p. 127 